\title{
IDEALS WITH SMALL AUTOMORPHISMS
}

\author{
BY WALTER RUDIN ${ }^{1}$
}

Communicated November 29, 1965

In [1], Forelli proves the following: If $G_{1}$ and $G_{2}$ are locally compact Abelian groups, if $J$ is a closed ideal in the group algebra $L^{1}\left(G_{1}\right)$, and if $\Psi$ is a homomorphism of $J$ into the measure algebra $M\left(G_{2}\right)$ with $\|\Psi\|=1$, then $\Psi$ is induced by an affine map of a coset in $\Gamma_{2}$ into $\Gamma_{1}$. (See $[1]$ for a more detailed statement. For notation and terminology, see [1] or [2]; $\Gamma_{i}$ denotes the dual group of $G_{i}$; the circle group will be denoted by $T$.) As Forelli points out in [1], the assumption $\|\Psi\|=1$ cannot be entirely discarded.

Actually, the assumption $\|\Psi\|=1$ cannot even be replaced by $\|\Psi\|$ $<1+\epsilon$, no matter how small $\epsilon>0$ is, even if "affine" is replaced by "piecervise affine" in the conclusion, and even if $G_{1}=G_{2}=T$ and $\Psi$ is assumed to be one-to-one.

Since the integer group $Z$ admits only countably many piecewise affine maps, the preceding statement is a consequence of the theorem below. By way of contrast, it may be of interest to mention that if $\Psi$ is a homomorphism of all of $L^{1}\left(G_{1}\right)$ into $M\left(G_{2}\right)$ and if $\|\Psi\|>1$, then $\|\Psi\| \geqq \sqrt{ } 5 / 2$ [2, p. 88].

Theorem. Suppose $0<\epsilon<1$. Let $E$ be a set of positive integers $\lambda_{k}$ such that $\lambda_{1}=1$ and

$$
\sum_{k=1}^{\infty} \frac{\lambda_{k}}{\lambda_{k+1}}<\frac{\epsilon}{6 \pi} .
$$

Let $J$ be the set of all $f \in L^{1}(T)$ whose nth Fourier coefficient $\hat{f}(n)$ is 0 for all $n$ not in $E$. Then $J$ is a closed ideal in $L^{1}(T)$, with continuum many automorphisms, and every automorphism $A$ of $J$ (other than the identity) satisfies the inequality

$$
1<\|A\|<1+\epsilon .
$$

We shall sketch the proof.

Each $A$ is induced by a permutation $\alpha$ of $E$. The gaps in $E$ show that no affine map (other than the identity) carries $E$ onto $E$. Thus $\|A\|>1$ if $A \neq I$.

We write $e(t)$ in place of $e^{2 \pi i t}$.

Let $\alpha$ be any permutation of $Z^{+}$(the positive integers), let

\footnotetext{
${ }^{1}$ Supported by NSF Grant GP-3483.
} 


$$
f(t)=\sum c(k) e\left(\lambda_{k} t\right), \quad g(t)=\sum c(\alpha(k)) e\left(\lambda_{k} t\right)
$$

be trigonometric polynomials in $J$. The theorem is an immediate consequence of the inequality

$$
\|g\|_{1} \leqq\left(1+\frac{7 \epsilon}{9}\right)\|f\|_{1} .
$$

To prove (4), fix $N$ so that $\hat{f}$ and $\hat{g}$ have their supports in $\left\{\lambda_{1}, \cdots, \lambda_{N}\right\}$. For $0 \leqq t<1$, let $D_{t}$ be the set of all $x=\left(x_{1}, \cdots, x_{N}\right)$ in $R^{N}$ such that

$$
\left\{\begin{array}{l}
\lambda_{k} t \leqq x_{k}<\lambda_{k} t+\lambda_{k} / \lambda_{k+1} \quad(1 \leqq k \leqq N-1), \\
\lambda_{N} t=x_{N}
\end{array}\right.
$$

and let $Q$ be the union of these $(N-1)$-cells $D_{t}$. We claim that $Q$ contains no point of $Z^{N}$, except 0 : Assume, to get a contradiction, that $x \in Q \cap Z^{N}, x \neq 0$.

If $x_{1}=0$, (5) implies $t=0$, hence $x_{2}=\cdots=x_{N}=0$. So $x_{1}=1=\lambda_{1}$. If $2 \leqq k \leqq N$ and $x_{k-1}=\lambda_{k-1}$, (5) gives

$$
\lambda_{k-1}<\lambda_{k-1} t+\lambda_{k-1} / \lambda_{k} \leqq \lambda_{k-1}\left(1+x_{k}\right) / \lambda_{k},
$$

or $\lambda_{k}<1+x_{k}$. Since $x_{k} \leqq \lambda_{k}$, we have $x_{k}=\lambda_{k}$. This leads to $x_{N}=\lambda_{N}$, a contradiction to the last equation (5).

Since $Q$ is a parallelopiped with one vertex at 0 it now follows that no two points of $Q$ are congruent modulo $Z^{N}$. Also, $Q$ has volume 1 . Thus if we regard functions on the torus $T^{N}$ as periodic functions on $R^{N}$, with period 1 in each of the variables $x_{1}, \cdots, x_{N}$, integration over $T^{N}$ may be replaced by integration over $Q$.

We return to our polynomials (3) and define

(6) $\quad F(x)=\sum_{1}^{N} c(k) e\left(x_{k}\right), \quad G(x)=\sum_{1}^{N} c(\alpha(k)) e\left(x_{k}\right) \quad\left(x \in R^{N}\right)$.

These are trigonometric polynomials on $T^{N}$. Clearly

$$
\|F\|_{1}=\|G\|_{1} \text {. }
$$

For $x \in D_{t}$, put $\widetilde{F}(x)=f(t), \widetilde{G}(x)=g(t)$. This defines $\widetilde{F}$ on $Q$, hence on $T^{N} ;(5)$ and (1) imply that

$$
\begin{aligned}
|\tilde{F}(x)-F(x)| & \leqq \sum_{1}^{N}|c(k)|\left|e\left(\lambda_{k} t\right)-e\left(x_{k}\right)\right| \\
& \leqq 2 \pi \sum_{1}^{N}|c(k)| \lambda_{k} / \lambda_{k+1} \leqq \frac{\epsilon}{3}\|f\|_{1}
\end{aligned}
$$


if $x \in D_{t}$. Since $\|\widetilde{F}\|_{1}$ can be computed by integrating $\mid \widetilde{F} \|$ over $Q$, the definition of $\tilde{F}$ shows, via Fubini's theorem, that $\|\tilde{F}\|_{1}=\|f\|_{1}$. By (8) this gives

$$
\|F\|_{1} \leqq\left(1+\frac{\epsilon}{3}\right)\|f\|_{1}
$$

The inequality $\|g\|_{1} \leqq(1+\epsilon / 3)\|G\|_{1}$ is obtained in the same way; combined with (7) and (9) it yields (4).

REMARK. If $E=\left\{\lambda_{k}\right\}$ is as in the theorem, if $1 \leqq p \leqq \infty$, if $\sum c(k) e\left(\lambda_{k} t\right)$ is the Fourier series of some $f \in L^{p}(T)$, and if $\alpha$ is any permutation of $Z^{+}$, the above proof also shows that $\sum c(\alpha(k)) e\left(\lambda_{k} t\right)$ is the Fourier series of a function $g \in L^{p}(T)$, and that $\|g\|_{p} \leqq(1+\epsilon)\|f\|_{p}$.

\section{REFERENCES}

1. Frank Forelli, Homomorphisms of ideals in group algebras, Illinois J. Math. 9 (1965), 410-417.

2. Walter Rudin, Fourier analysis on groups, Interscience, New York, 1962.

UNIVERSITY OF WisCoNSIN 